\title{
Polymeric fibers with tunable properties: Lessons from spider silk
}

\author{
M. Elices, G.V. Guinea, J. Pérez-Rigueiro *, G.R. Plaza \\ Departanento de Ciencia de Materiales, ETSI Caminos, Canales y Puertos, Universidad Politécnica de Madrid, 28040 Madrid, Spain
}

\begin{abstract}
A B S T R A C T
Making artificial fibers inspired in spider silks is considered as one of the milestones in the field of biomimetics. The interest is usually justified by the outstanding tensile properties of natural fibers, but it is usually overlooked that spider silk is endowed with a number of related properties - supercontraction, recovery and the existence of a ground state - that impart the material with additional desirable features, such as the possibility of tuning its mechanical behaviour. In this work we present a review on the experimental analysis and significance of these properties, stressing the contributions of our research group to the field. It is also demonstrated how the knowledge gained in the basic study of the natural material has been essential for the improvement of the properties exhibited by artificially processed bio-inspired silk fibers.
\end{abstract}

Keywords:

Spider silk

Supercontraction

Regenerated silk

\section{Introduction: experimental evidence and biological significance of silk supercontraction}

Spider silk is renowned for its unique combination of high tensile strength and large strain at breaking, which results in the largest work to fracture of any material either natural or artificial [1]. However, it is very often overlooked that spider silk is endowed with another feature comparable in its singularicy to its outstanding tensile properties: the ability to tune its mechanical behaviour in a predictable and controllable way [2], as reflected by the possibility of obtaining fibers at will with any given tensile behaviour within the range of stress-strain curves exhibited by the material.

The analysis of this and the other related features revised in this work started with the assessment of an intriguing property of spider silk labelled as supercontraction. The term supercontraction describes the shrinkage - up to half its initial length - of silk fibers spun from the major ampullate silk gland (MAS) [3,4] of orb-web spiders when immersed in water or subjected to high relative humidicy environments with their ends unrestrained.

It was also found that supercontraction leads to another significant modification of the properties of spider silk fibers, since the tensile behaviour of supercontracted MAS fibers tested in water corresponds to an elastomer [5] as illustrated in Fig. 1. The elastomeric behaviour is characterized by an extremely low value of the elastic modulus at small deformations and a significant increase of stiffness at large deformations, in contrast with the behaviour of MAS fibers tested in air, either as spun or subjected to previous supercontraction and dried (Fig. 1).

The absence of a clear biological function for the changes induced in MAS fibers by supercontraction thrust an unfinished debate about

\footnotetext{
* Corresponding author.

E-mail address: jperez甲mater.upmes (J. Pérez-Rigueiro).
}

its role in the performance of the material. In parallel with its discovery, it was proposed that supercontraction might allow keeping the web caut under high humidicy conditions [3]. This interpretation was apparently questioned by measurements on the relaxation of stresses in MAS fibers under high relative humidicy conditions [6], that seemed to escablish the complete relaxation of supercontraction stresses in a period of minutes. Later studies [7,8], however, escablished that cypical relaxation times were in the range of hours, and stresses relaxed a relatively small percentage of their initial value. These results indicated that the role of supercontraction in tensioning the web under humid environments could not be refused on purely mechanical grounds.

Paradoxically, the main reason against this initial hypothesis arises from the widespread presence of supercontraction throughout the evolution of the lineage. Thus, it has been found that the tensile properties of MAS fibers spun by orb-web spiders evolved in a rather unexpected way for a period of over 100 million years [9]. Thus, despite strong arguments in favour of a large divergence in the properties of MAS fibers spun by different species [10], the stressstrain curves of MAS fibers have remained remarkably constant. As it is shown in Fig. 2, it has been found that the stress-strain curves of MAS fibers from orb-web weaving species can be grouped into patterns not related to phylogeny but, intriguingly, differences between groups are especially evident in the properties of supercontracted fibers [11]. These findings, however, did not shed light on the exact role of supercontraction.

\section{Supercontraction, recovery and the existence of a ground state for MAS fibers}

Most of the uncertainties referred to the behaviour of silks can be related to a lesser or greater extent with the lack of a detailed knowledge on most of the microstructural and hierarchical aspects of 


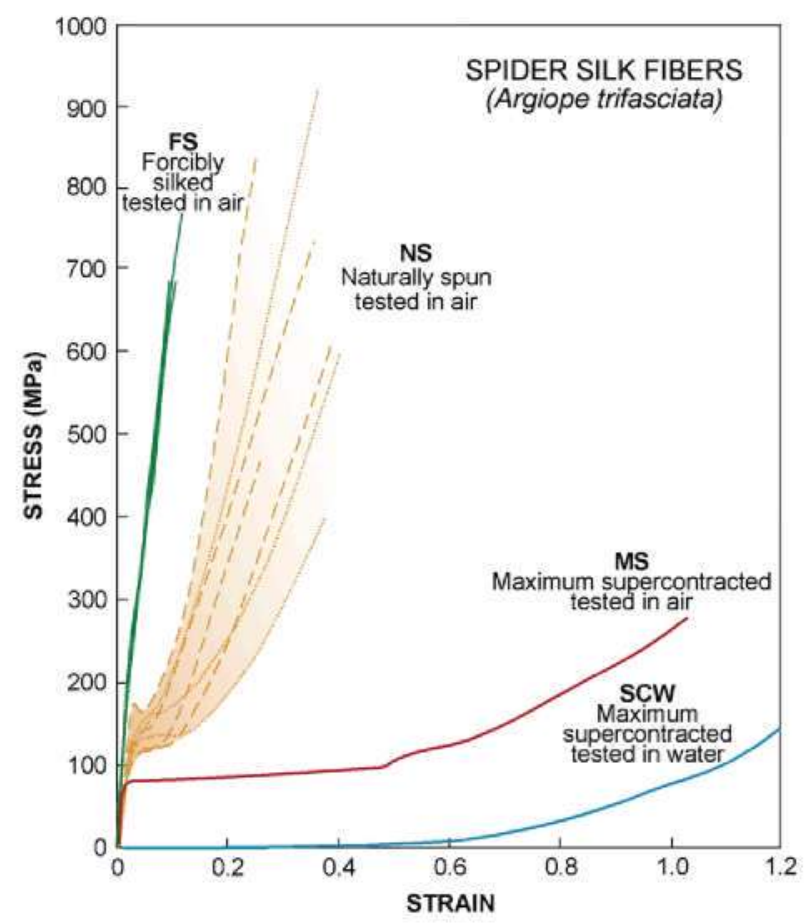

Fig. 1. Comparison of the stress-strain curves of MAS fibers obtained by forced silking (FS) $[53,54]$, naturally spun fibers as a safety line or for the structural elements of the web (NS), maximum supercontracted fibers tested in air (MS) and maximum supercontracted fibers tested in water (SCW). Forcibly silked (FS) fibers, correspond approximately to the stiffest condition of the material and maximum supercontracted (MS) fibers to the most compliant.

these fibers. In this regard, the identification of the elastomeric origin of the tensile properties of supercontracted fibers tested in water was a critical ingredient to one of the first and most successful attempts to model silk: the molecular (or Termonia's) model [12,13]. The molecular model assumes the existence of a double mesh composed of

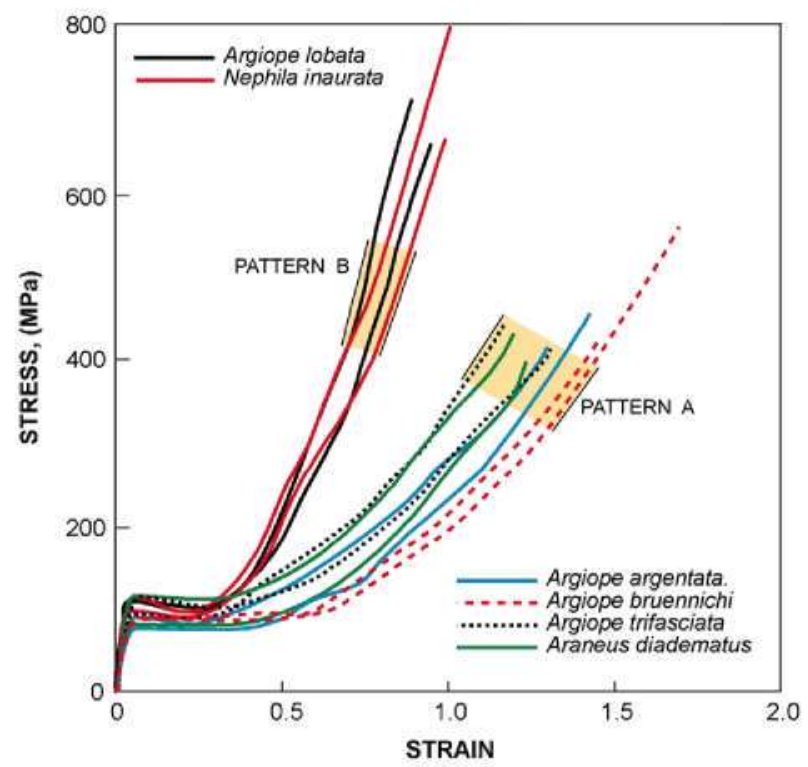

Fig. 2. Comparison of the stress-strain curves of maximum supercontracted fibers of six orb-web spider species: Argiope trifasciata, Argiope argentata, Argiope bruennichi, Argiope lobata, Araneus diadematus, and Nephila inaurata. These stress-strain curves group into two different patterns ( $A$ and $B$ ) that are not related to phylogeny, as shown by the species included in each pattern. $N$. inaurata diverged from the other species over 120 million years ago. polymeric chains with elastomeric behaviour and hydrogen bonds superimposed on them [14]. The elastomeric chains are linked through nanocrystals formed by piling up $\beta$-sheets $[15,16]$, whose formation depends on the sequence of the silk proteins $[17,18]$, and whose size is tuned to optimize simultaneously strength and resilience [19].

Supercontraction can be justified in the frame of the molecular model under the assumption that the polymeric chains are prestressed in the silk fibers spun by the spider. Immersion in water would cause the disruption of hydrogen bonds, since protein-protein interactions would be substituted by interactions between protein residues and free water molecules. The collapse of the hydrogen bonds mesh would allow the elastomeric chains to return to their equilibrium conformation by reducing the initial stresses which, in turn, would imply the reduction in the length of the fiber. The elastomeric behaviour of the fiber tested in water simply reflects the microscopic mechanical properties of its constituent protein chains in the absence of protein-protein hydrogen bonds.

Despite its success in justifying supercontraction and its satisfactory application to the numerical simulation of silk fibers, the model was not intended to give a complete description of the mechanisms responsible for the observed properties of silk. In this context, the full experimental confirmation that the main assumptions of the model corresponded to the actual behaviour of the material at molecular level was obtained from the analysis of an apparently independent aspect of spider silk.

Variability was soon acknowledged as one of the major drawbacks for the study of silks and, especially, spider silk [20,21]. It was found that fibers produced and retrieved under nominally identical conditions yielded stress-strain curves within an extremely wide range of values [3,22] (see Fig. 1), which in practice prevented the drawing of reliable conclusions on most aspects regarding the mechanical performance of the material [23]. From a biological perspective, however, the synthesis of a material with a fixed composition whose mechanical behaviour could be modified to adapt its properties to the immediate requirements of the spider seemed to offer an obvious advantage [24].

The analysis of the properties of supercontracted fibers and of the variability of MAS silk converged in the study of maximum supercontracted fibers, dried and tested in air, since it was established that the stress-strain curves of MAS fibers spun by Argiope trifasciata spiders concurred in a universal curve, which was labelled as maximum supercontracted or MS state [2]. It was later proven that the MS state constituted a true ground state, since any fiber could reach the MS state independent from its previous loading history $[25,26]$. This latter property was labelled as recovery since it was demonstrated that fibers stretched to large deformations, which yielded the irreversible modification of their tensile properties in air, recovered their initial properties after supercontraction (Fig. 3).

The existence of a ground state allows tuning the properties of MAS silk fibers in a predictable and reproducible way by simply following a given sequence of loading steps. Following this rationale a procedure, labelled as wet stretching [27] (Fig. 4), was developed that consists of stretching a silk fiber in water, which was previously subjected to maximum supercontraction. After the fiber reaches a predetermined length, their ends are fixed and the fiber is allowed to dry. The process is characterized quantitatively by the alignment parameter, $\alpha$, which is defined as $\alpha=\mathrm{L}_{C} / \mathrm{L}_{\mathrm{MS}}-1$, where $\mathrm{L}_{\mathrm{C}}$ is the length of the fiber after stretching in water and $\mathrm{L}_{\mathrm{MS}}$ is the length of the fiber after maximum supercontraction. As it is shown in Fig. 4 the whole range of tensile properties exhibited by MAS spider silk is obtained by simply varying the value of the alignment parameter. The initial studies on $A$. trifasciata MAS fibers were subsequently extended to other species, demonstrating the general character of the maximum supercontracted state and the recovery in the MAS silk of orb-web spinning spiders $[9,28]$. 

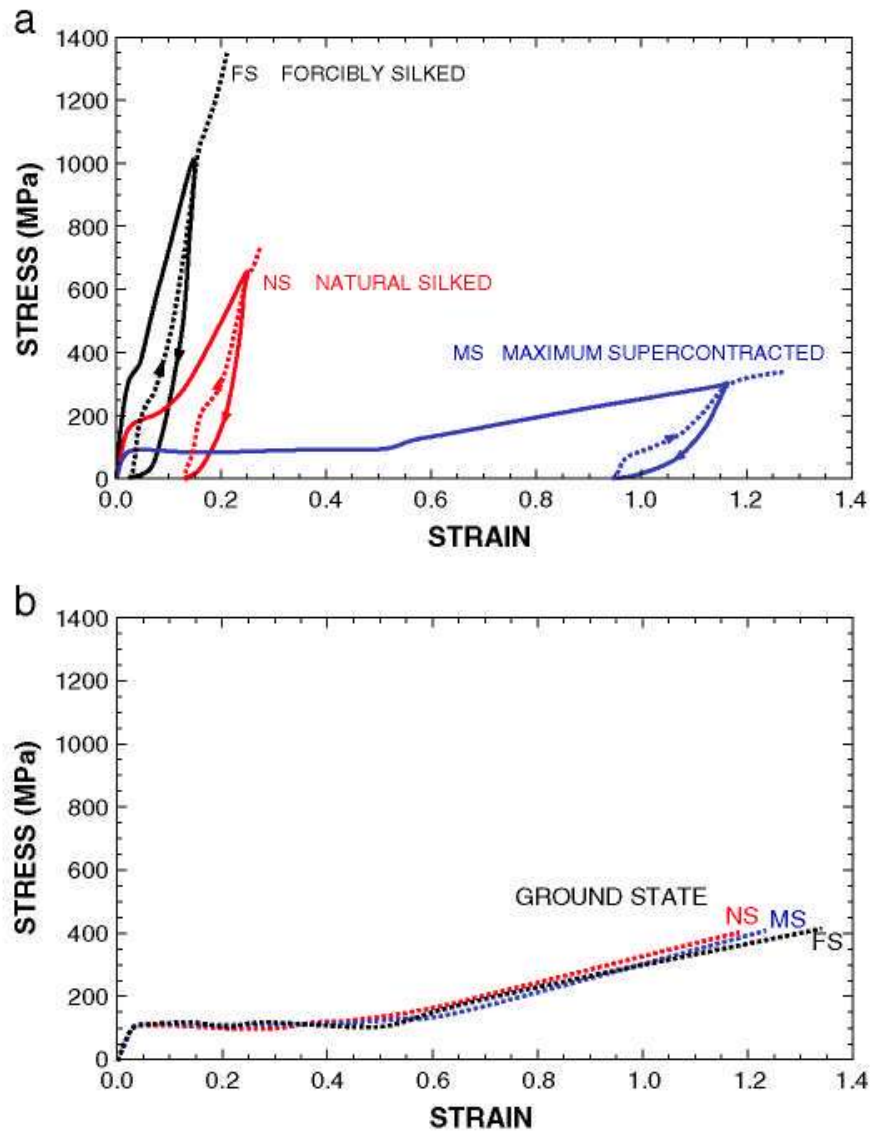

Fig. 3. Stress-strain curves of forcibly silked (FS), naturally spun (NS) and maximum supercontracted and dried (MS) fibers. a) Loading and unloading at high strains causes irreversible effects on the fibers as shown by the re-loading steps represented by dotted lines. b) Ground state reached by FS, NS and MS fibers after irreversible stretching and subsequent unrestrained immersion in water and drying. Notice that the stress-strain curves of all recovered fibers (from FS, NS and MS) concur.

It has been hypothesized that a similar mechanism takes place in the silk gland of the spider, being the basis of the production of natural fibers with tunable properties. Under this hypothesis, it is assumed that, immediately after solidification within the gland, the fiber shows the elastomeric behaviour of a supercontracted fiber in water. The spider can fix the alignment parameter by controlling the stress exerted on the elastomeric fiber during spinning [29].

The results presented above highlight the importance of the existence of a ground state, a property that underlies the shrinkage of the fibers in humid environments, their recovery after irreversible stretching and the tuning of the mechanical properties of MAS silk. Besides, the proposed relationship between supercontraction, processing and the possibility of modifying the tensile properties of the fibers sets the question of the biological function of supercontraction under a new perspective. In this regard, supercontraction itself would not be the main property that has driven natural selection during the evolution of orb-web spiders, but most likely the possibility of adapting the material to the requirements of the spider in the web or as a safety line [9]. In this context, supercontraction can be understood as a byproduct of the existence of a ground state, a hypothesis that does not exclude that tensioning the web under humid environments might be an additional benefit from the underlying property.

\section{Recovery in the context of biomimetics}

The production of artificial fibers inspired in spider silk is usually considered as one of the milestones in the field of biomimetics [30]. The relatively simple composition of the fibers and their excellent
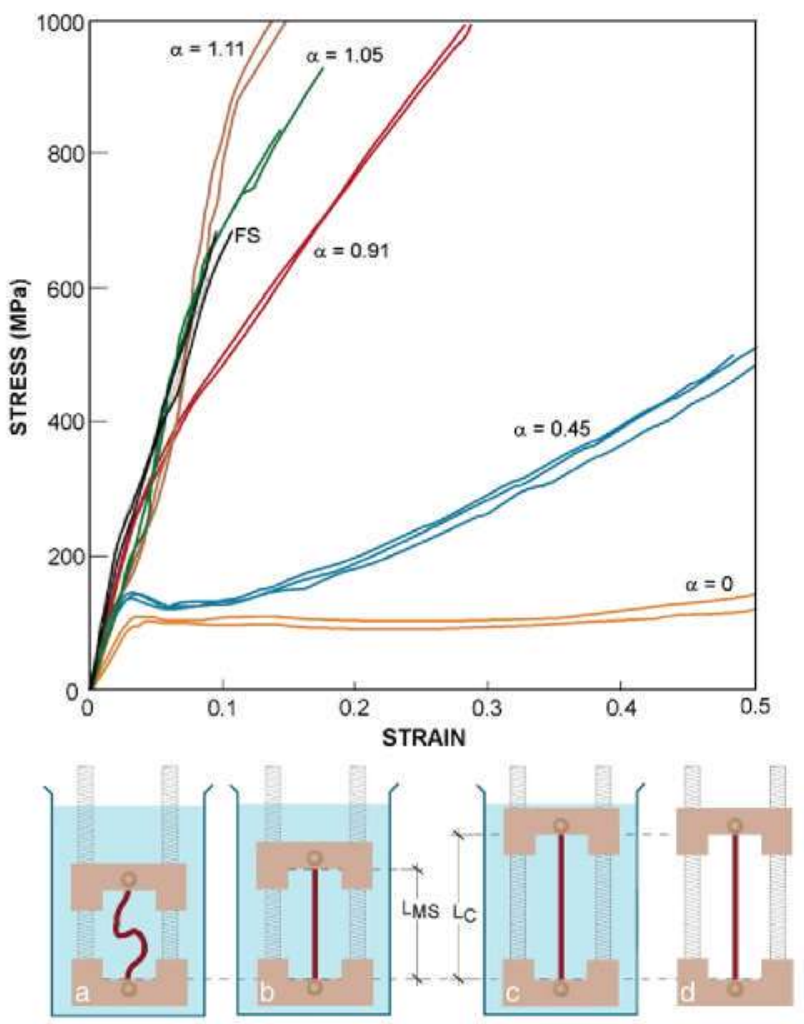

Fig. 4. Wet stretching procedure. The fiber is allowed to fully supercontract in water (a) down to its maximum supercontracted length $\mathrm{L}_{\mathrm{MS}}$ (b), and subsequently stretched in water up to a length $\mathrm{L}_{\mathrm{C}}$ (c). Water is removed and the fiber is allowed to dry overnight with their ends fixed (d). The evolution of the stress-strain curves with the alignment parameter is shown for different values of $\alpha$, ranging from $\alpha=0$ to $\alpha=1.1$. The alignment parameter $\alpha=0$ corresponds to the maximum supercontracted state. Forcibly silked fibers correspond to an alignment parameter of approximately $\alpha=1.1$.

tensile properties has led to an increasing number of works dealing both with the synthesis of proteins using genetic engineering techniques [31-33] and with the spinning of fibers from natural protein solutions [34,35]. The whole process was first completed in 2002 [36] with the production of completely artificial fibers bioinspired in MAS silk. However, the differences at all levels between natural and artificial processing paths pose the pressing question of which properties are maintained and which are lost in the artificial materials compared with those characteristic of the original ones.

In this regard, the previous discussion shows that supercontraction and related effects in MAS silk represent an adequate case study on the conditions required to mimic nature, due to its singularity and to the interest in its transfer to artificial fibers. Besides, it can also provide useful information in the debate on the relative importance of primary structure (i.e. sequence) and processing in the properties of silk fibers.

The sequence of the proteins that compose spider silk or spidroin has usually been considered as the main candidate to explain the outstanding behaviour of the material. The strict conservation along evolution for over 100 million years [18] of a small number of motifs of sequence [37] led to the evident conclusion that this repetition of amino acids was essential for the tensile behaviour of the fibers. This basic idea was supported by the remarkable differences between the mechanical properties of spider silk and silkworm silk [38]: Silkworm silk fibers are stiffer, but do not reach the high values of strain at breaking and tensile strength characteristic of spider silk. Even more significantly, silkworm silk fibers do not supercontract if immersed in water [1]. The absence of some characteristic motifs of sequence found in MAS spider silk and the appearance of a different motif in the sequence of silkworm silk fibroin [39] were considered as a clear 
evidence of the essential role played by the primary structure of the proteins.

However, the refinement of the spinning techniques for the synthesis of regenerated silkworm silk fibers has led to a substantial readdressing of the problem. Regenerated silkworm silk fibers are produced by dissolving natural silkworm silk, preparing a spinnable protein solution (dope) and synthesising the fibers through a wet spinning process [40]. Several processes were proposed following this basic scheme that differ in the use of different compositions for the dope [41-43]. However, the poor tensile properties reported for regenerated fibers did not allow inferring any reliable conclusion on the range of properties that could be reached through different processing paths from the same protein sequence. The modest performance of regenerated fibers compared with that of natural silkworm silk fiber was attributed by some researchers to the necessity of more sophisticated processing techniques that would somehow parallel the spinning process within the silk gland [44]. Other authors [45] still rely on the composition of the dope, as artificial silk only mimics part of natural silk proteins.

However, it was precisely the relevant role played by water in the performance of spider silk fibers, which led to the introduction of a very simple modification in the usual spinning procedure. Based on previous studies that highlighted both the importance of a postspinning drawing step [46], and on the improvement of the tensile properties of regenerated fibers after being stretched in water [47], the post-spinning drawing step was modified with the introduction of a water bath [48]. The modified procedure, labelled as immersion post-spinning drawing (IPSD), leads to regenerated fibers that show a ground state, as it was proven by the recovery of their properties after irreversible deformation if subjected to supercontraction in water (Fig. 5). Besides, the mechanical properties of the IPSD regenerated fibers are comparable to those measured from natural silkworm silk

\section{IMMERSION POST-SPINNING DRAWING (IPSD) PROCESS}
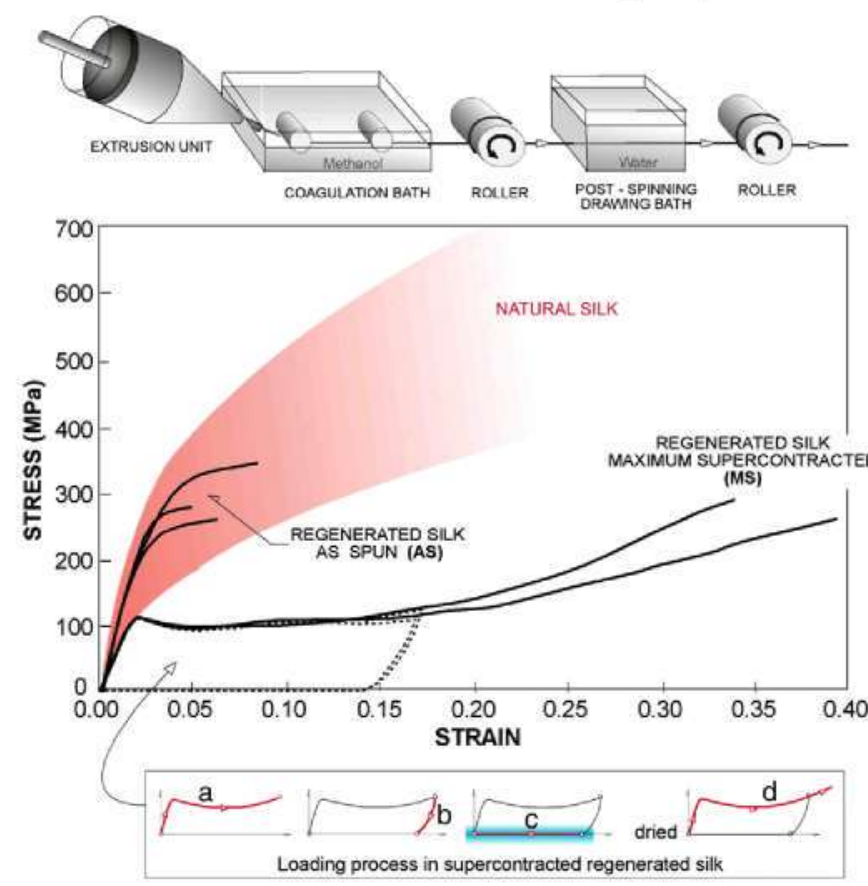

Fig. 5. Scheme of the spinning process of regenerated immersion post-spinning drawn (IPSD) fibers, and stress-strain curves of IPSD fibers. IPSD under the as-spun condition (AS) and after maximum supercontraction (MS) have been tested. The range of tensile properties exhibited by natural silkworm (Bombyx mori) silk fibers is also shown. Maximum supercontracted IPSD have been subjected to recovery tests as shown in the inset (a) loading in air, (b) unloading, (c) immersion in water with ends unrestrained and (d) drying. The dotted lines in the stress-strain curve of the MS fibers correspond to the loading-unloading part of the test in air. fibers in terms of work to fracture, although MS regenerated fibers show lower tensile strength and larger deformation at breaking. The existence of a ground state in IPSD fibers offers the first opportunity to tune the tensile properties of artificially processed fibers with a procedure that parallels that of natural spider silk.

\section{Future prospects}

IPSD fibers represent a significant breakthrough in the field of biomimetics, since it states clearly that the properties behind biological systems are more accessible than previously expected.

In this regard, it is apparent that there may be several compositions that yield similar microstructures under appropriate processing conditions which, in turn, results in comparable mechanical properties. Thus, it has been found that the microstructural parameters of IPSD silkworm fibers resemble more closely those of spider silk than those of natural silkworm silk, with which it shares a common sequence. The parallelism is exemplified by the analysis of the crystalline fraction in IPSD, natural silkworm silk and spider silk fibers. The crystalline fraction of IPSD fibers yielded a value of $20 \%$ from X-ray diffraction data, a value comparable with the value determined for MAS spider silk (10-15\%) and approximately one third of the value determined for natural silkworm silk (56-65\%).

Reciprocally, the same composition may generate materials with different behaviour depending on the spinning conditions, as it was first shown in natural spider silk. The extreme ability of MAS fibers to modify their properties, as stated from mechanical tests, was supported by a number of microstructural characterization techniques $[49,50]$ that addressed at least the broad details of the conformational changes related with the modification of the properties. The assessment that this functional flexibility is also shared by artificially processed regenerated fibers has also found support from microstructural characterization techniques [34,51].

This flexibility and the hidden surprises in the development of bioinspired materials can be illustrated with the effect exerted by post-spinning drawing on regenerated fibers. Post-spinning drawing in air was shown to lead to a significant improvement of the tensile properties of regenerated silkworm fibers, since the strain at breaking of post-spinning drawn fibers was comparable to that of the natural material [46]. It was immediately assumed that post-spinning drawing would modify the microstructure of regenerated fibers by generating a microstructure more similar to that of the natural material. However, the analysis of the fibers by atomic force microscopy showed that the typical size of the microstructural details of fibers subjected to post-spinning drawing was not only smaller than that observed in the natural material, but even smaller than that observed in fibers not subjected to post-spinning drawing [52]. Consequently, the improvement in the tensile behaviour was not the result of a microstructure more similar to natural silkworm silk, but rather the opposite.

The implications of these assessments could be far reaching for the development of biomimetics, since they give precedence to the need for a deeper understanding of the principles that underlie biological materials, over the attempt of copying nature by producing systems as similar as possible to the originals.

\section{Acknowledgements}

The authors are grateful to José Miguel Martínez for his help with the artwork. The work was funded by the Ministerio de Educación y Ciencia (Spain) through project MAT 2009-10258. The financial support from the Fundación Marcelino Botín and from the Comunidad de Madrid (Spain) to carry out this investigation through the MADR.IB$\mathrm{CM} / \mathrm{S}-\mathrm{SAL} / 0312 / 2006$ program is gratefully acknowledged. 


\section{References}

[1] D.L Kaplan, S. Lombardi, WS. Muller, S.A. Fossey, Novel Materials from Biological Sources, Stockton Press, New York, 1991.

[2] J. Perez-Rigueiro, M. Elices, G.V. Guinea, Polymer 44 (2003) 3733.

[3] R.W. Work, Text. Res. J. 47 (1977) 650

[4] F. Vollrath, Sci. Am. 266 (1992) 70.

[5] J.M. Gosline, M.W. Denny, M.E. Demont, Nature 309 (1984) 551.

[6] F.I. Bell, I.J. McEwen, C. Viney, Nature 416 (2002) 37.

17] K.N. Savage, P.A. Guerette, J.M. Gosline, Biomacromolecules 5 (2004) 675.

[8] G.V. Guined, M. Elices, J. Perez-Rigueiro, G. Plazd, Polymer 44 (2003) 5785.

[9] M. Elices, G.R Plaza, M.A. Arnedo, J. Pérez-Rigueiro, F.G. Torres, G.V. Guinea, Biomacromolecules 10 (2009) 1904.

[10] K.M. Rudall, W. Kenclington, Annu. Rev. Entomol. (1971) 73.

[11] K.N. Savage, J.M. Gosline, J. Exp. Biol. 211 (2008) 1937.

[12] Y. Termonia, in: M. Elices (Ed.), Structural Biological Materials, Pergamon Press, Amsterdam, 2000, p. 335.

[13] Y. Termonia, Macromolecules 27 (1994) 7378

[14] J.M. Gosline, M.E. Demont, M.W. Demly, Endeavour 10 (1986) 37.

[15] J.O. Warwicker, J. Mol. Biol. 2 (1960) 350.

[16] C. Riekel, F. Vollrath, Int ]. Biol. Macromol. 29 (2001) 203.

[17] R.E. Marsh, R.B. Corey, L. Pauling. Biochim. Biophys. Acta 16 (1955) 1.

[18] J. Gatesy, C. Hayashi, D. Motriuk, J. Woods, R. Lewis, Science 291 (2001) 2603

[19] S. Keten, Z.P. Xu, B. Ille, M.J. Buehler, Nat. Mater, 9 (2010) 359.

[20] MA. Garrido, M. Elices, C. Viney, J. Perez-Rigueiro, Polymer 43 (2002) 4495.

[21] R. Work, Text Res. J. 46 (1976) 485.

[22] J. Perez-Riguero, M. Elices, J. Llorca, C. Viney. J. Appl. Polym. Sci. 82 (2001) 2245

[23] D.L. Dunaway, B.L. Thiel, C. Viney, J. Appl. Polym. Sci. 58 (1995) 675.

[24] B. Madsen, 2.Z. Shao, F. Vollrath, Int. J. Biol. Macromol. 24 (1999) 301.

[25] M. Elices, J. Perez-Rigueiro, G. Plaza, G.V. Guinea, J. Appl. Polym. Sci. 92 (2004) 3537.

[26] M. Elices, J. Perez-Rigueiro, G.R. Plaza, G.V. Guinea, JOM 57 (2005) 60

[27] G.V. Guinea, M. Elices, J. Perez-Rigueiro, G.R. Plaza, J. Exp. Biol. 208 (2005) 25.

[28] Y. Liu, Z.Z. Shao, F. Vollrath, Nat. Mater, 4 (2005) 901.

[29] J. Perez-Rigueiro, M. Elices, G. Plaza, J.I. Real, G.V. Guinea, J. Exp. Biol. 208 (2005) 2633.

[30] R.V. Lewis, Chem. Rev, 106 (2006) 3762.

[31] J.T. Prince, K.P. Mcgrath, C.M. Digirolamo, D.L Kaplan, Biochemistry (N.Y.) 34 (1995) 10879

[32] Y. Fukushima, Biopolymers 45 (1998) 269.

[33] D. Huemmerich, T. Scheibel, F. Vollrath, S. Cohen, U. Gat, S. Ittah, Curr. Biol. 14 (2004) 2070.

[34] K.A. Trabbic, P. Yager, Macromolecules 31 (1998) 462.

[35] O. Liivak, A. Blye, N. Shal,, L.W. Jelinski, Maciomolecules 31 (1998) 2947.
|36| A. Lazalis, S. Arcidiacolk, Y. Huang. J.F. Zhou, F. Duguay, N. Chretien, E.A. Welsl, J.W. Soares, C.N. Karatzas, Science 295 (2002) 472.

37| M. Xu. R.V. Lewis, Proc. Natl Acad. Sci. USA 87 (1990) 7120.

[38] J. Perez-Rigueiro, C Viney, J. Llocca, M. Elices, J. Appl, Polym. 5 ci. 70 (1908) 2439.

[39] OY. Xia, Z.Y. Zhou, C. Lu, DJ Cheng, F.Y. Dai, B. Li, P. Zhao, X.F. Zha, T.C. Cheng, C.L. Clad, G.Q Pan, J.S. Xu, C. Liu, Y. Lin, J.F, Qian, Y. Hou, Z.L. Wu, G.R. Li, M.H. Pan, C.F. Li, Y.H. Shen, X.Q. Lan, L.W. Yuan, T. Li, H.F. Xu, G.W. Yang, Y.J. Wan, Y. Zhu, M.D. Yu, W.D. Shen, D.Y. Wu, Z.H. Xiang, J. Yu, J. Wang, R.O Li, J.P. Shi, H. Li, G.Y. Li, J.N. Su, X.L. Wang, G.Q Li, Z.J. Zlhang, Q.F. Wu, J. Li, Q.P. Zhang, N. Wei, J.Z. Xu, H.B. Sun, L. Dong, D.Y. Liu, S.L. Zhao, X.L Zhao, Q.S. Meng, F.D. Lan, XG. Huang, YZ. Li, L. Fang, C.F. Li, D.W. Li, Y.Q Sun, Z.P. Zhang, Z. Yang, Y.Q. Huang, Y. Xi, Q.H. Oj, D.D. He, H.Y. Huang, X.W. Zhang, Z.Q. Wang. W.J. Li, Y.Z. Cao, Y.P. Yu, H. Yu, J.H. Li, J.H. Ye, H. Chen, Y. Zhou, B. Liu, J. Wang, J. Ye, H. Ji, S.T. Li, P.X. Ni, J.G. Zhang, Y. Zhang. H.K. Zheng, B.Y, Mao, W. Wang, C. Ye, S.G. Li, J. Wang, G.K.S. Wong, H.M. Yang. Science 306 (2004) 1937

|40| K.K.Chawla, Fibrous Materials, Cambridge University Press, Cambridge, U.K. 1998.

41] K. Matsumoto, H. Uejima, T. Iwasaki, Y. Sano, H. Sumino, J. Appl. Polym. Sci. 60 (1996) 503

42| E. Marsano, P. Corsini, C. Arosio, A. Boschi, M. Mormino, G. Freddi, Int. J. Biol. Macromol. 37 (2005) 179.

43) S.W. Ha, A.E. Tonelli, S.M. Hudson, Bionacronolecules 6 (2005) 1722.

[44] S. Rammensee, U. Slotta, T. Scheibel, A.R. Bausch, Proc. Natl Acad. Sci. USA 105 (2008) 6590 .

|45| C.N. Karatzas, N. Chretien, F. Duguay, A. Bellemare, J.F. Zhou, A. Rodenhiser, S.A. Islam, C. Turcotte, Y. Huang, A. Lazaris, High-toughness spider silk fibers spun from soluble recombinant silk produced in mammalian cells, Bioteclnology of Biopolymers, Steinbüchel and Doi, Wiley-VCH, Weinheim (Germany), 2005, p. 945.

46| P. Corsini, J. Perez-Rigueiro, G.V. Guinea, G.R. Plazd, M. Elices, E. Marsano, M.M. Carnasciali, G. Freddi, J. Polym. Sci. B: Polym. Phys. 45 (2007) 2568.

|47| G.R. Plaza, P. Corsini, J. Perez-Rigueiro, E. Marsano, G.V. Guinea, M. Elices, J. Appl. Polym. Sci. 109 (2008) 1793

[48] G.R. Plaza, P. Corsini, E. Marsano, J. Perez-Rigueiro, L. Biancotto, M. Elices, C. Riekel, F. Agullo-Rueda, E Gallardo, J.M. Calleja, G.V. Guinea, Macrolnolecules 42 (2009) 8977 .

|49| J. van Beek, J. Kuinmerlen, F, Vollrath, B. Meier, Int. J. Biol. Maciomol. 24 (1999) 173.

[50] Z. Shao, F. Vollrath, J. Sirichaisit, RJ. Young, Polymer 40 (1999) 2493.

151] F. Xie, H.H. Zliang, H.L. Shao, X.C. Hu, Int. J. Biol. Macromol. 38 (2006) 284

[52] J. Perez-Rigueiro, L. Biancotto, P. Corsini, E. Marsano, M. Elices, G.R. Plaza, G.V. Guinea, Int. J. Biol. Macromol. 44 (2009) 195.

[53] RW. Work, P.D. Emerson, J. Arachnol. 10 (1982) 1.

[54] G.V. Guinea, M. Elices, J.I. Real, S. Gutierrez, J. Perez-Rigueiro, J. Exp. Zool. A Comp. Exp. Biol. 303A (2005) 37. 